\title{
An Explanation About Canonical Forms and Real Molecule in Resonance Theory, through Instructors Own Sketches Analogy
}

Faiz Ahmed*

*Department of Chemistry, Government College University Faisalabad, 38000 Faisalabad, Pakistan.

\section{ABSTRACT}

In elementary chemistry courses students often demonstrate difficulty with real understanding of Resonance Theory i.e. canonical structure vs. real molecule difference, so unanswered puerile questions during lecture made the subject boring. In such situations use of suitable analogy other than the subject area make the teaching more effective. Using an analogy from the daily life act as a powerful tool to explain curious questions efficiently to develop the interest of the students in subject. Sharing of personal experiences and analogies among scientific community is an effective way to spread scientific knowledge magnificently.

\section{GRAPHICAL ABSTRACT}

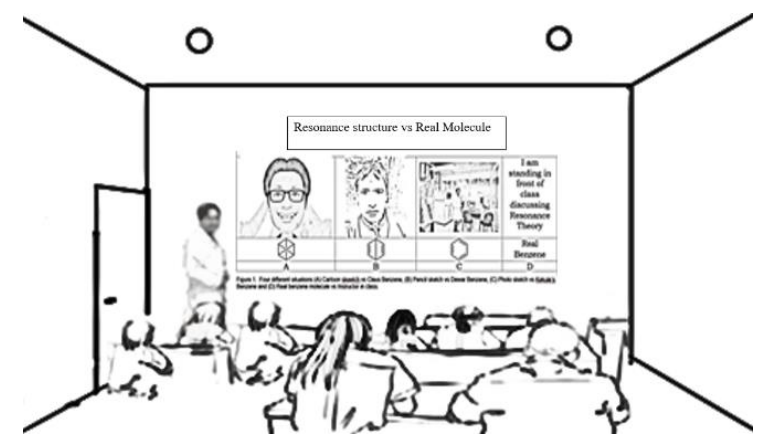

KEYWORDS

Sketches Analogy, Canonical forms, Real molecule, Resonance Theory, Chemical Pedagogy

In organic chemistry during basic concepts lectures, students often demonstrate difficulty with the principle of resonance. ${ }^{1}$ Simple questions in undergraduates chemistry class sometime create 
an environment which force the teacher to think out of the box to explain these questions using analogy $y^{2}$ in problem other than the subject i.e. Wheland enthusiast of resonance theory in his book ${ }^{3}$ about resonance phenomena used the analogy of mule which is a hybrid between a horse and a donkey, ${ }^{3}$ in an attempt to explain the theories of intermediate stages and of mesomerism picture of the benzene molecule which is a hybrid moiety, not identical with either of the Kekulé's structure or proposed canonical forms but intermediate between them. ${ }^{4}$ Although resonance theory is one of the most important constituent of structural chemistry, ${ }^{5}$ still highly useful and important section of the pedagogy of organic chemistry concepts ${ }^{6}$ however in some situations this use of imaginary institution to describe real molecules was incompatible with the dialectical materialism. ${ }^{3,4,7}$ Similar type of problem faced by the organic chemist while explaining the structure of organic compounds especially the canonical forms and the actual molecule while

discussing the Rules of resonance. ${ }^{8}$ During basic chemistry courses all over the world a small fraction of students raise puerile questions during the lecture, which are ignored by the teachers sometime to save the lecture time and utility on more important concepts as a result that small fraction of students find chemistry as boring subject, where imaginary things are discussed and taught. However suitable analogy related to the query or topic make it easy, understandable and develop students interest in the subject ${ }^{9-14}$ i.e. cartoon characters analogy for resonance by $\mathrm{R}$. Starkey. ${ }^{14}$

\section{Resonance principle, Canonical Structure Vs. Real Molecule:}

During my 15 years teaching experience in organic chemistry I also meet with students every year who find difficult to understand such lines "All canonical forms do not contribute equally to the true molecule and it is more stable than all these canonical forms $"$ and they immediately raise the questions, what is real molecule and why we are unable to draw its exact structure? In start of my career I also used Wheland analogy ${ }^{4}$ however students curiosity remain unsatisfied, sometime 
few raise objection that mule, donkey and horse are three different animals and all has existence, while here we are discussing only one molecule that is benzene. After the flash of these questions more students join that small fraction of students with doubts. To face such situation I developed following questions their answers and analogies.

Question: Class can we represent a student on identity card by writing his name, registration number on national data base and photo?

\section{Class Answer: Yes}

Question: In chemistry how we can represent a chemical substance?

Class Answer: Through its name, chemical formula, CAS Registry Number or draw its structure to discuss it.(Sometime I also participate in this answer)

Next we discussed about organic compounds simple chemical formula from which different structures can be deduced i.e. ethanol and dimethyl ether both have same chemical formula $\mathrm{C}_{2} \mathrm{H}_{6} \mathrm{O}$, to overcome this issue organic chemist prefer to represent each compound with localized bonding systems through Lewis structure, while compounds with delocalized bonding system are represented through different canonical forms using Resonance theory or method.

\section{Sketch Analogy:}

After these question answers and discussion I draw few sketches about myself on white/black board or show my photos or emojis on screen according to the situation along with different structure of benzene proposed by different scientists (See Figure 1).

After showing Figure 1, I ask the students following questions:

Question: Is column A, B and C photos in instructor's sketch row represents me?

Answer: Yes.

Question: In current situation which photo/sketch represents me most and which on least? 
Answer: In instructors sketch row, photo in column $\mathbf{C}$ represents me more while $\mathbf{A}$ represents least.

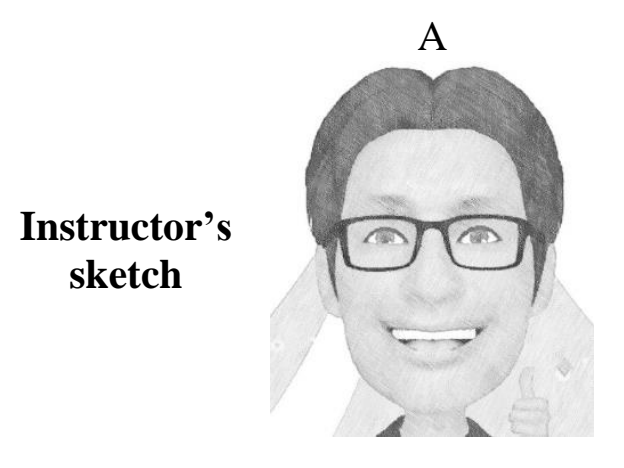

\section{Benzene sketch}
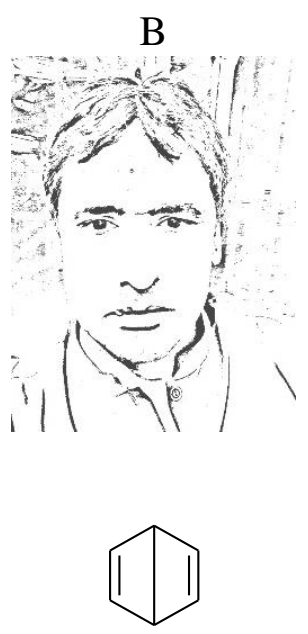

$\mathrm{C}$
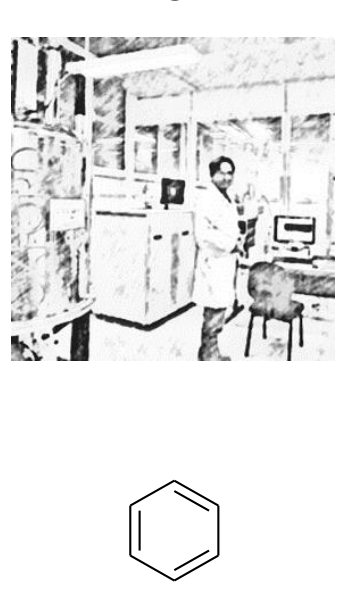

D

I am standing in front of class discussing Resonance Theory

Real benzene molecule is in reagent bottle on the desk.

Figure 1. Four different situations (A) Cartoon sketch vs. Claus Benzene, (B) Pencil sketch vs. Dewar Benzene, $(\mathbf{C})$ Photo sketch vs. Kekule's Benzene and (D) Real benzene molecule vs. Instructor in class.

Then I continue that even $\mathbf{C}$ does not represent me completely because it only represent my side which is in front of camera, it also does not move as I am moving in front of whole class, which create fourth situation D my real existence. Due to limitations of two dimensional sketch on paper it is not possible to create a duplicate copy of a person on a paper. Similarly, same case is with benzene canonical structure in column $\mathbf{A}, \mathbf{B}$ and $\mathbf{C}$, however these canonical structures provide us an opportunity to discuss a molecule on a paper, on board or in a book. Although we are unable to draw a real benzene molecule which is actually in the reagent bottle on the desk which also creates fourth situation $\mathbf{D}$, however we can draw its different possible sketches to represent and discuss it. After this analogy I ask whole class did you understand the canonical forms and a real molecule. They say yes and have no further doubts. We can also use any other living organism sketches i.e. dog, cat etc. as an example, however instructor's sketches and presence in front of class act as an influential instrument for students understanding toward the 
difference between canonical structure and real molecule. Thus a simple analogy act as a powerful tool to develop the interest of students in subject with better elucidation, so there is need to share more such personal experiences and analogies to spread scientific knowledge more effectively all around the world.

\section{AUTHOR INFORMATION}

Corresponding Author

*E-mail: faiz79goraya@gmail.com, faizahmed@gcuf.edu.pk

\section{ACKNOWLEDGMENTS}

My mentors Prof. Dr Abdul Rauf and Prof. Ashfaq Mahmood Qureshi who’s mentorship enabled me to learn chemistry and to all my students who raise thought provoking questions during lecture.

\section{REFERENCES}

1. T. Kim, L. K. Wright and K. Miller, Chemistry Education Research and Practice, 2019, 20, 659-666.

2. V. M. Shahani and J. Jenkinson, Chemistry Education Research and Practice, 2016, 17, 417-428.

3. G. W. Wheland, The Theory of Resonance and its Application to Organic Chemistry, 1944.

4. E. T. Strom, in Pioneers of Quantum Chemistry, American Chemical Society, 2013, vol. 1122, ch. 3, pp. 75-115.

5. L. C. Pauling, Proceedings of the Royal Society of London. A. Mathematical and Physical Sciences, 1977, 356, 433-441.

6. W. C. Herndon, Journal of the American Chemical Society, 1973, 95, 2404-2406.

7. $\quad$ G. W. Wheland, Advanced Organic Chemistry, 1948.

8. in March's Advanced Organic Chemistry, 2006, DOI: https://doi.org/10.1002/9780470084960.ch2, pp. 32-105.

9. J. E. Cortés-Figueroa, W. I. Pérez, J. R. López and D. A. Moore-Russo, J. Chem. Educ., $2011, \mathbf{8 8}, 932$.

10. M. Orgill, G. Bodner, N. Pienta, M. Cooper and T. Greenbowe, Chemists' Guide to Effective Teaching, 2005.

11. J. Orvis, D. Sturges, S. Rhodes, K.-J. White, T. W. Maurer and S. M. Landge, Journal of Chemical Education, 2016, 93, 879-885.

12. T. P. Silverstein, J. Chem. Educ., 1999, 76, 206.

13. S. Spezzini, Int. J. Scholarship Teach. Learn., 2010, 4, 1.

14. R. Starkey, J. Chem. Educ., 1995, 72, 542. 